To appear in Proceedings of the 43rd Hawai'i International Conference on the System Sciences (HICSS-43), January, 2010, Kauai, Hawai'i (CD-ROM). New Brunswick: Institute of Electrical and Electronics Engineers, Inc. (IEEE).

\title{
The Temporal Development of Representational Practices: Implications for Theory and Analysis of Situated Learning
}

\author{
Daniel Suthers and Richard Medina \\ Department of Information and Computer Sciences, University of Hawai ${ }^{\top} i$, USA \\ (suthers|rmedina\}@hawaii.edu
}

\begin{abstract}
Technological resources for collaborative learning are productively conceived of as resources appropriated by learners as they develop their competencies. Therefore, to understand the implications of technological designs for collaborative learning, it is necessary to examine learners' practices over time. Microanalytic approaches are most suitable for understanding learners' practices, but have traditionally been applied primarily to single small episodes of learning. This limitation is related to an unnecessarily strict interpretation of the temporal locality of situated action. Drawing on a prior analysis of the temporal development of practices through which inscriptions become representations in online mathematics problem solving, this paper generalizes microanalysis to include temporally prior episodes in which interactionally relevant resources and practices are constructed. Related theoretical points concerning the relationship between the concept of practices and ethnomethodological principles of relevance and contingency are discussed.
\end{abstract}

\section{Introduction}

To understand how the design of technology environments influence (or fail to influence) collaborative learning, we must understand how the resources of those environments are appropriated for and thereby influence the course of the activity that produces learning. Following Suthers [46] and the thinkers on which he draws, we question the adequacy of two common simplified views of the role of technology in collaborative (or other forms of group) learning. First, although "computer mediated communication" enables people to interact with each other, it is not sufficient to treat technology merely as a channel of communication that substitutes for face-to-face interaction in ways that are often considered to be inadequate $[4,8,33,39]$. We might find ways to go "beyond being there" [7, 15, 37], considering the unique opportunities offered by the medium and engaging in different kinds of interaction.
Second, although technology can be used to prompt and guide learners through automated tutors $[1,6]$ or scripts $[10,16]$, it is not just a medium for controlling learners, and in particular not limited to influencing immediate action. Learners can appropriate the resources of the technological medium for their own purposes, and this appropriation (as well as the influence of the technology) can develop over time.

The authors accept that various technologies enable and guide communication in certain ways, but place an equal emphasis on the agency of those who use the technologies. Collaborative learning is not merely a matter of communication or information sharing, but also involves knowledge-construction phenomena that take place at the group level $[38,40]$. A given technology offers affordances (action potentials) that may influence how learners engage in this knowledge construction [23, 24, 47, 50], but not in a deterministic manner. Participants in collaborative learning will develop practices by which they use the technology, and these practices may emerge over time. Thus, if we want to understand the implications of our technological designs for learning, we need to examine learners' practices over time.

Analyses based on coding individual acts and then performing statistical analyses on these aggregations often obscure the sequential practices through which collaboration takes place and appropriates material affordances [48, 49]. Other methodological traditions are stronger in the study of practice. Microanalytic approaches such as interaction analysis [17], and ethnomethodology and conversation analysis $[5,12]$ as applied to the learning sciences $[21,22]$ can provide analysts with a rich understanding of the meaning-making activities of learner-participants and how the resources of the environment figure in these activities.

Yet such methods are not without problems. Elsewhere we are addressing the problem that interaction analysis does not easily scale up to larger data sets $[49,48]$. In this paper we focus on a related limitation: in practice, microanalysis has been applied only to short transcripts, identifying interactional relationships within temporally bounded segments of 
interaction, yet effective design requires that we understand the sequential development of practices that appropriate technological resources over time. This particular restriction is not a necessity: "micro" can mean looking closely at interaction rather than putting on temporal blinders. We call into question assumptions (discussed below) to the effect that the "situation" (and hence the analytically relevant data) is limited to recent history or bounded by a recording or transcript selected for analysis. The primary practical contribution of this paper is a strategy for extending microanalysis back to prior episodes, by identifying references participants make and resources they draw on that are contingent on their shared prior history of activity. The strategy is motivated by and illustrated with an analysis we conducted that shows how a group's learning accomplishment drew upon prior interaction through which inscriptions became representational resources for the group. In order to understand participants' accomplishments in a given session, we used a two-level analytic approach that found relevant prior episodes through persistent inscriptions and participants' references, and then applied microanalysis within each episode to uncover the representational practices that were developed and enacted in those episodes.

The particular way in which we extend microanalysis back in time (as well as the fact we are doing so) is motivated by a particular interpretation of situated cognition and learning $[3,43,26]$ and ethnomethodological relevance $[11,21]$ that is offered as the primary theoretical contribution of this paper. It requires some background to appreciate. Theories of situated cognition and learning were motivated in part by a reaction against cognitive explanations of behavior that postulate mental representations and mutual beliefs as determinants of behavior [43]. Neither mental representations nor "common ground" [31] are visible to the analyst or participants, and such accounts have been critiqued on this basis, e.g., [3, 19, 43]. Instead, situated accounts look to the structure of the physical environment (especially cultural artifacts) and social interactions, resulting in (we believe) a bias towards accounts that rely on elements visible in a given situation, and hence towards uncritical acceptance of reliance on short transcripts.

Another influence came from a renegade branch of sociology known as ethnomethodology [11]. Ethnomethodology rejects explanations of social order that postulate external forces acting causally on social actors. Instead, ethnomethodology (literally, the study of the methods of an ethnos-not a research methodology), holds that the methods by which participants make sense of their activity as rational and accountable to each other are precisely the same as the means by which that activity is organized. Hence, ethnomethodological relevance states that the only relevant analytic accounts are those that identify what participants demonstrably use or orient towards in organizing their actions [21]. This has given rise to perceptions of ethnomethodology as being anti-theoretical [18]. Ethnomethodology aligns with situated cognition in the principle of contingently achieved accomplishments, which states that actor's accomplishments are deeply contingent upon the socially produced immediate situation, and hence meaning can only be understood in that sequential context [21].

The theoretical work of this paper derives out of a tension that authors perceived between temporally local interpretations of the ideas just discussed and the temporally extended nature of ideas such as recurring practices and methods. This was not just a theoretical debate: we also wanted to understand how analysts' movements back into time via a transcript (something that participants cannot do in real time) could be ethnomethodologically relevant. This paper discusses two resolutions. First, abstractions such as "strategies" underlying participants' practices are understood as resources for accountable organization of action rather than determining structures. Second, the temporal scope of the current situation is expanded by identifying ways in which prior resources are available to and invoked by participants. These more nuanced theoretical positions are present elsewhere in the literature, but are brought together here with an analytic approach to offer a better way towards advances in technology for learning.

The paper is organized as follows. First, we present a motivating example, summarizing an analysis we previously conducted that shows how a group's accomplishment is temporally extended across sessions through the re-enactment of representational practices. Then we generalize our method of tracing practices over time via contingencies between acts. Finally, we discuss the theoretical and methodological issues raised in the introduction, focusing particularly on the above-mentioned resolutions.

\section{Understanding Group Accomplishments as Historically Situated}

In this section we summarize an analysis detailed in $[27,30]$ of the work of students in the Virtual Math Teams (VMT) Spring Fest 2006, based on data provided by Gerry Stahl and also described in [42]. Our analysis

- Identified some representational practices employed by participants in the sessions being analyzed. 
- Showed that the inscriptions employed in these practices become representations for the group's problem solving through participants' interactions.

- Showed that the group's problem solving drew upon their prior practices as resources, i.e., their group accomplishment is historically situated, and that persistent inscriptions play a role in enabling this temporal extension of group cognition.

Our present concern is not with the specific results of that analysis, but rather with theoretical and methodological issues relevant to "methods and frameworks for studying the practices of producing understanding within online environments" [20].

\subsection{Virtual Math Teams}

Virtual Math Teams [42] is a special project of the Math Forum at Drexel [34], a long-standing online community and resource for mathematics education. In typical VMT sessions, several student participants at distributed locations interact using ConcertChat [32; see Figures 1-3] to solve mathematics problems. ConcertChat is a software environment consisting of a shared whiteboard and a chat tool with the capability of referencing the whiteboard in a linked approach to artifact-centered discussion [45]. Participants also used a wiki to post their solutions during and after each session. These wiki pages, the software log files and re-playable instances of activity logged in the ConcertChat environment served as our data sources. The replayer provides a rich contextual view useful for understanding the participants' inscriptional work as it developed concurrently with the interaction in the chat tool.

The data we analyzed was derived from a VMT project involving three student teams $(\mathrm{A}, \mathrm{B}, \mathrm{C})$ each consisting of three student participants and one moderator, all at different geographic locations. Our analysis focuses on the work of team B. During the course of the project, each team convened in four separate sessions to work on algebraic geometry problems. We analyzed the first three sessions; a relevant analysis of the fourth is found in [41]. For the convenience of those who wish to relate the present discussion to our full analysis in [27], we use four digit numbers in angle brackets (e.g., <4009>) to refer to transcripts in [27], or to time stamps in the original data not published in that paper.

\subsection{A Group Accomplishment}

In the third of four VMT sessions that took place on four separate days, the students are trying to derive a formula for counting the squares in a diamond shaped tiling pattern (Figure 3). At one point, the lower left inscription in Figure 3 is present, but the colored inscription lower right has not yet been drawn. Aznx (a self-selected pseudonym) says, "I think I have an interesting way to look at this problem" $<4009>$, and briefly describes an innovative representation of the problem at hand. This representation enables decomposition of the problem into mathematically simpler sub-problems, one of which was solved in the first day's session.

Aznx alludes to the problem decomposition and its relationship to prior work only very briefly $<19: 35: 45-19: 36: 21>$. Yet, Aznx's partner Bwang indicates that he understands <4067>, supplies the actual visualization of the problem representation (the colored figure lower right of Figure 3), and verbally summarizes the problem decomposition that it supports $<4096>$.

We undertook our analysis to explore this event in comparison to Stahl's [41] definition of group cognition as thought-like processes that take place through group interaction. The definition implies that a group must interact each time it "cogitates" about a given problem, but the amount of work visible in the immediate episode of interaction does not seem sufficient to account for the complexity of problem solving being accomplished by the group. We wanted to find where the work was being done. It was not sufficient to assume the work was done in Aznx's mind alone, because that would not explain how the others were able to appropriate the solution so quickly. But the methods of problem representation and decomposition that the group applied were not locally produced in this session. The group was implicitly drawing on prior work. We were interested in the tension between this historicity and the ethnomethodological focus on group accomplishments as being "performed/achieved locally in the circumstances of their production" [21]. This tension is key to understanding the theoretical points of this paper.

\subsection{The Development of Representational Practices}

Working back to identify relevant episodes in prior sessions using methods to be described in the next section, Medina et al. [27, 30] traced the development of a problem solving strategy, which we termed problem decomposition, and three endogenous representational practices through which it was realized, which we termed inscribe-first, solve second, visualize decomposition, and modulate perspective. Problem decomposition is the common problem solving strategy of breaking a complex problem down into simpler components such that the solutions can be 
recomposed. Inscribe-first, solve second is the practice of generating inscriptions (e.g., diagrams or formulas) that show the structure of the problem before solving it. Visualize decomposition is the closely related strategy of using these inscriptions to make the problem decomposition visible. Finally, modulate perspective is the practice of providing alternative representational points of view while negotiating agreement on the nature of the problem.

The strategy of problem decomposition and the practices of inscribing before solving and visualizing the decomposition with these inscriptions all appeared in Session 1, where participants were trying to count the sticks in a staircase-shaped figure of squares. Early in the session, Bwang said "you can divide the thing into parts" $<0182>$, and then visualized this decomposition by drawing horizontal and vertical lines spatially separated (Figure 1).

On a subsequent day, in Session 2, participants are tasked with inventing a figure of their own conception for analysis. Quicksilver has proposed a "pyramid." This proposal is originally understood by one participant as vertically flat pyramid, and by another participant as a horizontally flat board. Some interactional work is required before the group agrees on what sort of pyramid is meant, settling on a pyramid that is three-dimensional. In the process, a new practice, that of modulating or offering different perspectives on a figure, is introduced, with four alternate visualizations (two of which are shown in figure 2). Prior practices of visualizing the problem decomposition before constructing a formula are also continued, but the decomposition is visualized using color rather than spatial separation, showing that the decomposition practice is a generalized idea or abstraction rather than being tied to a specific inscriptional realization.

All of these practices resurface in Session 3 (Figure 3). Participants are discussing ways to decompose the problem into layers by which the diamond figure grows. They are inscribing representations of proposed decompositions before working out the formulaic solution. Figure 3 shows two ways to decompose the problem using two different inscriptional devices: drawing a line and coloring a portion. After the left hand visualization was drawn, Aznx suggested that they compute the area of the bounding square and then subtract the corners, which have the same form as the staircase figures solved in Session 1. Bwang's rapid appropriation of Axnx's "interesting way to look at this problem" resulted in the right hand visualization and evidenced the availability of prior practices to the group.

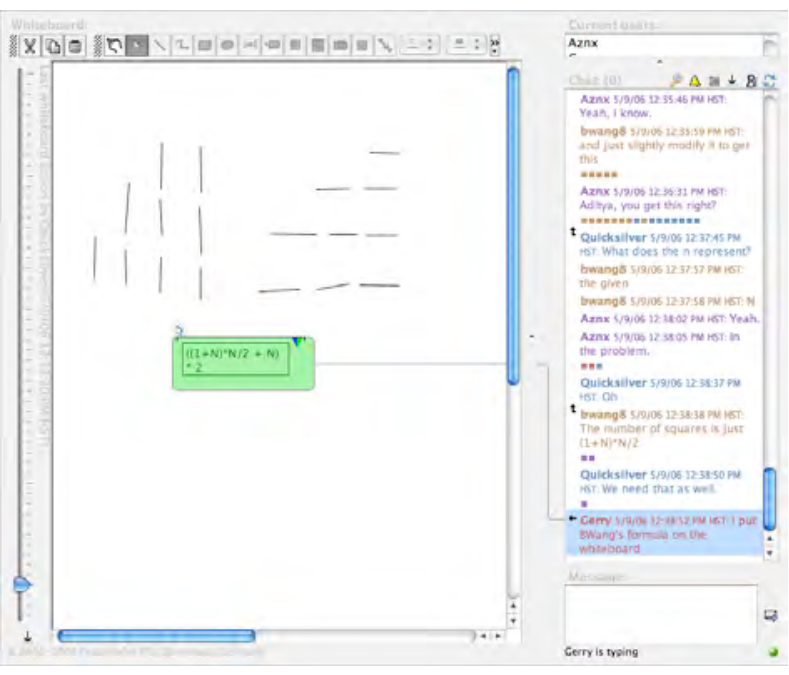

Figure 1. Initial appearance of practices in Session 1

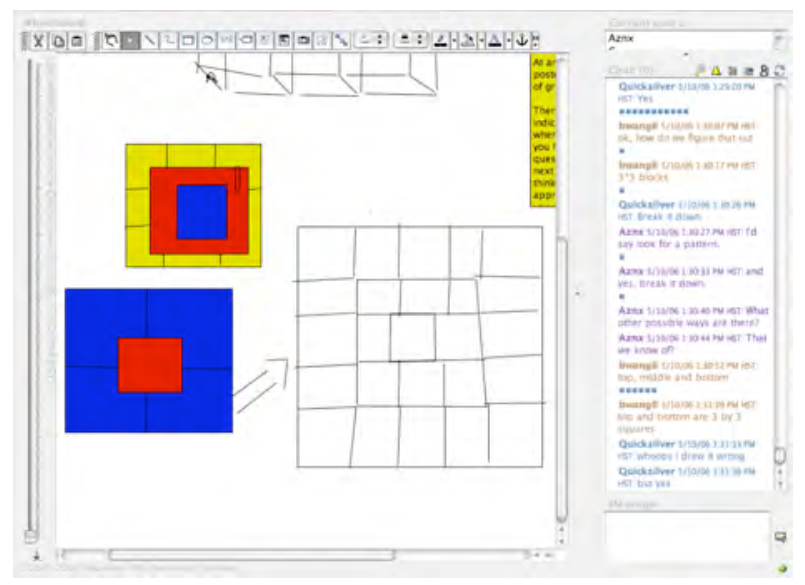

Figure 2. Using color to visualize decompositions in Session 2

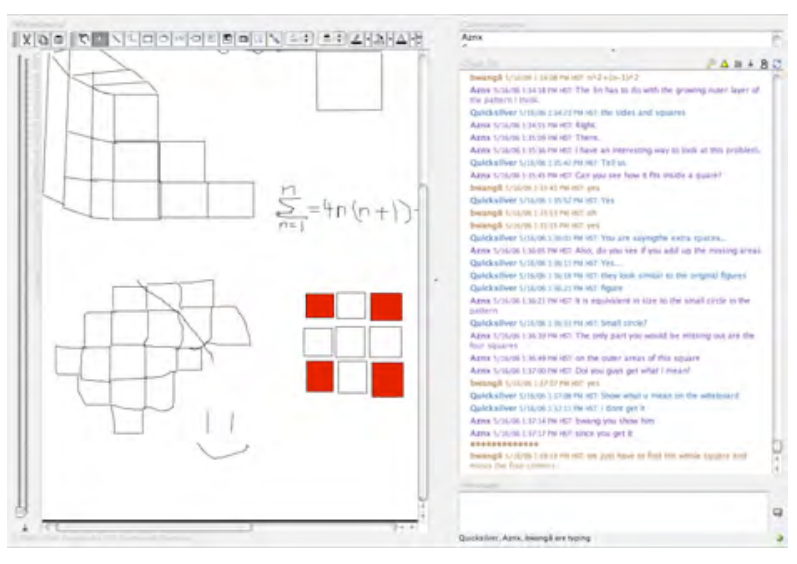

Figure 3. Reapplying practices in Session 3 


\subsection{Discussion}

The resolution of how participants were able to quickly appropriate Aznx's insight in Session 3 is straightforward: members of a group can draw on their prior interactions and on the products of those interactions as resources, and so need not work out their methods anew each time as they engage in group cognition. That learners draw upon prior experience is well known, but microanalytic research has tended to focus on short isolated transcripts and there are tensions with theoretical traditions outlined in the introduction to this paper. The analysis showed that the "interesting way of looking" is understood quickly by participants because of their shared history. Similarly, we as analysts need to understand their activity in context of their history. To sufficiently account for participants' actions one must look back in time, and identify ways in which the current episode is contingent upon prior episodes. We turn briefly to how we do this before discussing implications for theory.

\section{Summary of Analytic Approach}

Our method of analysis helps to uncover how the immediate intersubjective meaning-making of a group is contingent on prior episodes [29, 48, 49]. When an analyst encounters an episode that seems to draw upon resources beyond those that are immediately apparent, the method calls for a shift to a different level of analysis that seeks relevant prior episodes in which these resources are constructed, and then a return to microanalysis within these episodes to show the constructions. The method provides a way to select from a large record of prior interactions those episodes that are likely to be relevant.

\subsection{Uptake and Contingencies}

We distinguish two kinds of relationships between acts: "uptake" and "contingencies". Our ultimate interest is in uptake: the relation between two acts in which the chronologically later act takes up some aspect or product of the prior act as being relevant for present purposes. For example, participants in sessions 2 and 3 are taking up inscriptional and strategic practices developed in prior sessions, and within each session participants take up each others' prior contributions by replying to them or appropriating elements of them. But uptake is not always directly apparent and cannot be assumed: it must be demonstrated. The demonstration is grounded in observable relationships between two acts. Contingencies are the observable ways in which one act potentially draws upon another. Contingencies span the "many metaphysical shades between full causality and sheer inexistence" [25], and come in various forms. A reply to a message is contingent on the prior creation of that message. Inscriptional objects in a workspace may be subsequently elaborated. A contingency can also be observed when inscriptional configurations are reused. (Persistence is an important property of the VMT medium for the development of representational practices because it makes these practices meronymically available through their associated inscriptions.) Recurring lexical items is another form of contingency (e.g., $<0182>$ in Session 1: "you can divide the thing into parts" and $<1777>$ in Session 2: "divide them up into levels"). The most ubiquitous contingency is temporal contiguity: adjacency pairs are based on the observation that an utterance is contingent upon the immediately prior utterance, unless otherwise marked [36]. (Temporal contiguity is applied within the microanalysis of an episode rather than to find prior relevant episodes.)

\subsection{Two Levels of Analysis}

In our approach, an analysis begins with identification of an episode of interest, and then works both backwards and forwards at two levels (termed global and local for convenience of reference) to construct accounts of the participants' interaction and accomplishments.

We begin with microanalysis of an episode of interest, such as one in which learning or problem solving is displayed. For example, the Session 3 episode we analyzed was of interest due to the rapid application of Aznx's insight. The move to extend analysis to prior episodes is driven by the analytic need to understand a given episode. For example, we wanted to understand how the inscriptions used or referenced by participants in Session 3 offered representational resources for resolving the question at hand. Participants' own references (e.g., "divide into levels") or the resources they draw on (e.g., use of color to distinguish a portion of an inscription) direct the temporal extension. We follow participants' orientations in order to ensure that the analysis brings forth historical elements that are real for participants in the present (i.e., are ethnomethodologically relevant) rather than imposing analysts' postulated structures.

When the need to expand the scope of analysis is identified, work shifts to a global level of analysis to find chronologically prior episodes in which (for example) related inscriptions were constructed, in order to understand how they previously functioned as representations for the participants. We first identify the point where the development of the inscription in question had been completed, because this is where the 
inscription had reached the form in which it was available in future episodes. Then the temporal extent of the episode was defined by working back to where the construction and discussion of the inscription began as well as forward to the completion of discussion about the inscription. All forms of interaction are used to identify and delimit relevant episodes, since participants' chat referenced, labeled and interpreted inscriptions in the whiteboard. This process of searching backwards for relevant prior episodes was repeated until we had identified a chain back to the first session.

Then, the local level of analysis works forward within each episode to construct a microanalytic account of the interaction within that episode. Analysis at this level is undertaken in a manner similar to conversation analysis $[14,35]$ as it is applied in the field of computer supported collaborative learning [e.g., $22,41]$, but attends to inscriptional acts as well as conversations in the chat tool. Discussions in the chat are often woven with inscriptional work in the whiteboard in a manner that distributes conversation across the two media [44]. A trace of the contributions made in each of these media (chat and inscriptional acts) provide a resource for understanding contingent interaction. Certain events within each segment are annotated to document relationships between individual acts. For example, we document the introduction or reuse of inscriptional practices or linguistic references that demonstrate contingent relationships from one act to the next. During local analysis, the segment under consideration is sometimes expanded to encompass the episode of meaning-making relevant to the question at hand. Issues identified locally also initiate further global analysis of relationships between episodes, as described in the previous paragraph.

In summary, our analysis works forwards locally within each episode to identify participants' methods of meaning-making with the resources available; and when participants appear to orient towards nonlocal resources, we follow their references backwards to identify prior episodes on which a given episode's accomplishments may have been contingent. The result is a trace of contingencies at two granularities that enables us to recognize patterns in the data and better understand collaborative interaction and its accomplishment in shared environments [28, 48].

\section{Implications for Theory}

The analysis of $[27,30]$ identified several representational practices by which the problem solving practice of problem decomposition is realized: inscribe-first, solve second; modulate perspective; and visualize decomposition. Theoretical and analytic issues derive from the reapplication of practices. The term practice has multiple senses reflected in dictionary definitions. Many of these include a notion of repetition or recurrence, whether explicitly (e.g., a habitual or customary way of doing something; the repeated performance of an activity in order to perfect a skill) or implicitly (e.g., the exercise of an occupation or profession; the act or process of doing something). Similarly, a method is understood as a means or procedure for accomplishing or approaching something, especially a systematic or established one.

\subsection{Problematizing Practices, Relevance and Contingency}

The analysis demonstrated that the observed uses of inscriptions were practices by showing that each such use recurred and was enacted by multiple participants. The analysis demonstrated that they were distinct practices by showing that sometimes one was enacted without the others. Most important for the present discussion, it demonstrated that a given practice could recur in different manifestations. For example, "visualize decomposition" was first enacted in this data by Bwang in the first session, who said $<0182>$ "you can divide the thing into parts" and then drew horizontal and vertical lines separately. In the second session, after Bwang says $<1777>$ "divide them up into levels", Quicksilver uses color to visualize the decomposition of a pyramid into layers <1824-1882>. In the third session, Quicksilver asks Aznx to use coloring similarly to visualize the decomposition of a figure into the part that grows, but Aznx enacts this request by drawing a line $<3950>$, demonstrating that it is the decomposition, not the particular inscriptional device, that mattered to participants.

The practice of visualizing decompositions is enacted through three different inscriptional means in these examples. What is the "practice" that is being applied across these sessions? What is taken up when practices are reinvoked? Calling these diverse enactments the "same" practice implies that practices involve abstractions that persist over time. Then, "contingently-achieved accomplishments" are contingent upon something abstract and nonlocal that is yet accessible within "the circumstances of their production" [21]. The ethnomethodological policy of relevance accepts only those accounts of activity that participants themselves orient towards in that activity. Does postulating recurring practices as abstractions contradict ethnomethodological relevance, or do participants orient towards multiple enactments as being the same abstract practice? Is this nonlocality in the concept of "practice" incompatible with the policy 
of "contingently-achieved accomplishment" [21]? These two questions are addressed in the next sections.

\subsection{The Relevance of Strategies to Participants}

To address the first question above, the data shows that the practices we identified are not merely etic constructions. Participants orient towards strategies that are repeated, as evidenced by the following excerpts:

$<1459>$ "What can we use that we already know?"

$<1466>$ "I'd suggest yesterday's problem"

$<1473>>$ "using the formula from yesterday's problem"

They also display understanding that a given strategy can be enacted in different ways, as evidenced by the multiple manifestations of visualize decomposition that follow the suggestion to "divide":

$<0182>$ Bwang: "you can divide the thing into parts"; Bwang draws separate horizontal and vertical lines $<1777>$ Bwang: "divide them up into levels"; Quicksilver then uses color to visualize the decomposition of a pyramid into layers $<1824-1882>$

$<3950>$ Quicksilver: "color the portion"; Aznx replies by drawing a line

This does not imply that any recurring abstract patterns of activity are ethnomethodologically relevant practices. Care must be taken to identify strategies towards which participants are demonstrably oriented.

\subsection{The Temporal Extent of "Local" Circumstances}

There are two related facets of the apparent tension between practice and contingency to address: the role of abstractions such as strategies, and the temporal extent of the "local" circumstances of production. First, strategies are resources to be drawn on rather than deterministic structures [43]. Members who share a practice can recognize the contingently produced application of such strategies (and they do, as noted above). Its contingent production is what makes a strategy observable [9]; the strategy in turn provides an account (in the ethnomethodological sense) of the meaning of that production. Second, the apparent tension between actions as contingent on the present situation and practice as involving something from the past may derive from an artificial dichotomy in our understanding of the situation or context. Conversation analysis views action as simultaneously context-shaped and context renewing [12]. The history of interaction shapes the present in an obvious way through persistent artifacts, but also because historical ways of seeing and acting remain available to participants as part of this shaping context. Analysts may need to look back in time to uncover something we cannot otherwise see, but our epistemological necessity should not be confused with participants' reality. For participants, the current context is already constituted partly by their history, from which elements such as prior practices can be reinvoked without explicit description, for example, through metonymy such as an associated phrase, or meronymy such as reproducing an inscriptional portion of that practice.

\section{Conclusions}

Advances in technology-supported learning require that we understand how participants' learning accomplishments are contingent or draw on resources of the designed environment. As illustrated in this work, these contingencies are not temporally bounded by a transcript or session. Therefore, if microanalysis of practices is to be relevant to the practices of technology supported collaborative learning, then we must understand how participants' accomplishments are contingent or draw on temporally extended practices associated with the resources of the designed environment. We need to reconceptualize the "micro" in microanalysis to mean looking closely, but not necessarily looking only at a short segment of transcript. This is not a contradiction with the idea that learning accomplishments are "achieved locally in the circumstances of their production" if we reconceptualize the temporal extent of these "local circumstances." While analysts need to look back in time to uncover what is not present for the analyst, for participants the current context is partially constituted by their shared history, including prior practices and resources that are easily reinvoked.

This theoretical resolution leaves us with methodological problems: how we can identify recurring practices (particularly in the large data sets characteristic of online interaction) if their instantiations vary, and how we can identify prior accomplishments that are relevant to participants. A method for identifying interactionally relevant prior episodes by following contingencies was offered. The method is driven by analytically demonstrated need rather than by theory. It is invoked when participants appear to draw on resources beyond what is apparent in the local interaction. Devices by which aspects or products of prior interaction are re-invoked as relevant for present purposes were identified: these may include inscriptional configurations or historical and meronymic references. Similar elements are identified in prior sessions, and new transcripts for microanalysis 
are bounded by identifying the conversation about these elements. Microanalysis within episodes is then performed to identify the construction of resources and practices taken up in later sessions.

The method is similar to CN-ARE [2] in that it relies on the ability to identify and follow "tracers" or "identical elements" [51] over time. Workspace manipulations that revisit previously constructed inscriptions and literal recurrence of terms (e.g., "divide") make it easier to identify relevant prior episodes, but it may be more difficult to find recurring practices that vary more dramatically in their instantiations. We are seeking opportunities to explore these analytic issues further with other data.

Temporally extended microanalysis is particularly needed at present. There is perhaps an overemphasis on educational applications that use technologies primarily as means for temporally immediate interventions (e.g., coaching and scripting) to (re)enforce desired practices. Overemphasis on deterministic uses of technology [13] will neglect how learners' practices can develop through temporally extended meaning-making that appropriates technological resources.

\section{Acknowledgements}

We thank Ravi Vatrapu for comments on the analysis, and Gerry Stahl for providing VMT data.

\section{References}

[1] J. R. Anderson, A. T. Corbett, K. R. Koedinger and R. Pelletier, Cognitive tutors: Lessons learned, Journal of the Learning Sciences, 4 (1995), pp. 167-207.

[2] S. A. Barab, K. E. Hay and L. C. Yamagata-Lynch, Constructing networks of action-relevant episodes: An in situ research methodology, The Journal of the Learning Sciences, 10 (2001), pp. 63-112.

[3] W. J. Clancey, Situated Cognition: Stepping Out of Representational Flatland, AI Communications, 4 (1991), pp. 107-112.

[4] H. H. Clark and S. E. Brennan, Grounding in communication, in L. B. Resnick, J. M. Levine and S. D. Teasley, eds., Perspectives on Socially Shared Cognition, American Psychological Association, 1991, pp. 127-149.

[5] S. E. Clayman and D. W. Maynard, Ethnomethodology and conversation analysis, in P. t. Have and G. Psathas, eds., Situated Order: Studies in the Social Organization of Talk and Embodied Activities, International Instituted for Ethnomethodology and Conversation Analysis \& University Press of America, Washington, D.C., 1995, pp. $1-30$.

[6] A. T. Corbett, K. R. Koedinger and J. R. Anderson, Intelligent tutoring systems, in M. Helander, T. K. Landauer and P. Prabhu, eds., Handbook of Human-Computer Interaction, Elsevier Science B.V., Amsterdam, 1997, pp. 849-874.

[7] J. N. Cummings, T. Finholt, I. Foster, C. Kesselman and K. A. Lawrence, Beyond Being There: A Blueprint for Advancing the Design, Development and Evaluaiton of Virtual Organzations (Final report from workshops on building effective virtual organzations) 2008.

[8] R. L. Daft and R. H. Lengel, Information richness: a new aproach to managerial behavior and organizational design, in L. L. Cummings and B. M. Staw, eds., Research in Organizational Behavior 6, JAI Press, Homewood, IL, 1984, pp. 191-233.

[9] P. Dourish and G. Button, On "Technomethodology": Foundational Relationships Between Ethnomethodology and System Design, Human-Computer Interaction, 13 (1998), pp. 395-432.

[10] F. Fischer, I. Kollar, H. Mandl and J. M. Jaake, Scripting Computer-Supported Collaborative Learning: Cognitive, Computational and Educational Perspectives, Springer, New York, 2007.

[11] H. Garfinkel, Studies in Ethnomethodology, Prentice-Hall, Englewood Cliffs, New Jersey, 1967.

[12] C. Goodwin and J. Heritage, Conversation Analysis, Annual Review of Anthropology, 19 (1990), pp. 283-307.

[13] E. Hamilton and A. Feenberg, The technical codes of online education, E-Learning, 2 (2005), pp. 104-121.

[14] J. Heritage, Conversation analysis: Methodological Aspects, in U. Quasthoff, ed., Aspects of oral communication, Walter de Gruyter, Berlin, 1995, pp. 391-418.

[15] J. Hollan and S. Stornetta, Beyond Being There, CHI'92 Human Factors in Computing Systems, ACM/SIGCHI, 1992, pp. 119-125.

[16] P. Jermann and P. Dillenbourg, Elaborating new arguments through a CSCL script, in J. Andriessen, M. Baker and D. D. Suthers, eds., Arguing to Learn: Confronting Cognitions in Computer-Supported Collaborative Learning Environments, Kluwer, Dordrecht, 2003, pp. 205-226.

[17] B. Jordan and A. Henderson, Interaction Analysis: Foundations and practice, The Journal of the Learning Sciences, 4 (1995), pp. 39-103.

[18] V. Kaptelinin and B. A. Nardi, Acting With Technology: Activity Theory and Interaction Design, MIT Press, Cambridge, 2006.

[19] T. Koschmann, C. Goodwin, C. LeBaron and P. Feltovich, Dissecting Common Ground: Examining an Instance of Reference Repair, in J. D. Moore and K. Stenning, eds., Twenty-Third Annual Conference of the Cognitive Science Society, Lawrence Erlbaum Associates, 2001, pp. 516-521.

[20] T. Koschmann and G. Stahl, A comparative analysis of understanding practices in the VMT environment, 
in A. Dimitracopoulou, C. O'Malley, D. D. Suthers and P. Reimann, eds., Computer Supported Collaborative Learning 2009 Community Events Proceedings, LuLu, Rhodes, Greece, 2009, pp. 28-30.

[21] T. Koschmann, G. Stahl and A. Zemel, The Video Analyst's Manifesto (or The Implications of Garfinkel's Policies for Studying Practice within Design-Based Research), in R. Goldman, R. Pea, B. Barron and S. J. Derry, eds., Video Research in the Learning Sciences, Lawrence Erlbaum Associates, Inc., Mahwah, NJ, 2007.

[22] T. Koschmann, A. Zemel, M. Conlee-Stevens, N. Young, J. Robbs and A. Barnhart, How do people learn: Member's methods and communicative mediation, in $\mathrm{R}$. Bromme, F. W. Hesse and H. Spada, eds., Barriers and Biases in Computer-Mediated Knowledge Communication (and how they may be overcome), Kluwer Academic Press, Amsterdam, 2005, pp. 265-294.

[23] R. B. Kozma, The material features of multiple representations and their cognitive and social affordances for science understanding, Learning and Instruction, 13 (2003), pp. 205-226.

[24] S. P. Lajoie and S. J. Derry, eds., Computers as Cognitive Tools, Lawrence Erlbaum Associates, Hillsdale, NJ, 1993.

[25] B. Latour, Reassembing the Social: An Introduction to Actor-Network-Theory, Oxford University Press, New York, 2005.

[26] J. Lave and E. Wenger, Situated Learning: Legitimate Peripheral Participation, Cambridge University Press, Cambridge, 1991.

[27] R. Medina, D. Suthers and R. Vatrapu, Inscriptions becoming representations, in C. O'Malley, P. Reimann, D. Suthers and A. Dimitracopoulou, eds., Computer Supported Collaborative Learning Practices: CSCL 2009 Conference Proceedings, International Society of the Learning Sciences, Rhodes, Greece, 2009, pp. 18-27.

[28] R. Medina and D. D. Suthers, Bringing Representational Practice From Log to Light, International Conference for the Learning Sciences, Utrecht, 2008.

[29] R. Medina and D. D. Suthers, Using a contingency graph to discover representational practices in an online environment, Research and Practice in Technology Enhanced Learning, 4 (submitted).

[30] R. Medina, D. D. Suthers and R. Vatrapu, Representational practices in VMT, in G. Stahl, ed., Studying Virtual Math Teams, MIT Press, Cambridge, MA, in press, pp. tbd.

[31] A. Monk, Common Ground in Electronically Mediated Communication: Clark's Theory of Language use., in J. M. Carroll, ed., HCI Models, Theories and Frameworks: Towards a Multidisiplinary Science., Mogan Kaufmann, San Francisco, 2003, pp. 265-289.

[32] M. Mühlpfordt and M. Wessner, Explicit referencing In chat supports collaborative learning, in $\mathrm{T}$.
Koschmann, D. D. Suthers and T.-W. Chan, eds., Computer Supported Collaborative Learning: The Next 10 Years!, Lawrence Erlbaum Associates, Mahwah, NJ, 2005, pp. 460-469.

[33] G. M. Olson and J. S. Olson, Distance matters, Human-Computer Interaction, 15 (2000).

[34] K. A. Renninger and W. Shumar, The Centrality of Culture and Commuinity to Participant Learning at and with the Math Forum, in K. A. Renninger and W. Shumar, eds., Buidilng Virtual Communities, Cambridge University Press, Cambridge, 2002.

[35] H. Sacks, Lectures on Conversation, Blackwell, Oxford, UK, 1992.

[36] H. Sacks, E. A. Schegloff and G. Jefferson, $A$ simplest systematics for the organization of turn-taking for conversation, Language, 50 (1974), pp. 696-735.

[37] A. Salovaara, Inventing new uses for tools: A cognitive foundation for studies on appropriation, Human Technology, 4 (2008), pp. 209-228.

[38] M. Scardamalia and C. Bereiter, Higher Levels of Agency for Children in Knowledge Building: A Challenge for the Design of New Knowledge Media, The Journal of the Learning Sciences, 1 (1991), pp. 37-68.

[39] J. Short, E. Williams and B. Christie, The Social Psychology of Telecommunications, John Wiley and Sons, London, 1976.

[40] G. Stahl, Group Cognition: Computer Support for Collaborative Knowledge Building, MIT Press, Cambridge, MA, 2006.

[41] G. Stahl, Meaning making in CSCL: Conditions and preconditions for cognitive processes by groups, in $\mathrm{C}$. Chinn, G. Erkens and S. Puntambekar, eds., The Computer Supported Collaborative Learning (CSCL) Conference 2007, International Society of the Learning Sciences, New Brunswick, NJ, 2007.

[42] G. Stahl, Studying Virtual Math Teams, MIT Press, Cambridge, MA, in press.

[43] L. A. Suchman, Plans and Situated Actions: The problem of human-machine communication, Cambridge University Press, Cambridge, 1987.

[44] D. D. Suthers, $A$ qualitative analysis of collaborative knowledge construction through shared representations Research and Practice in Technology Enhanced Learning, 1 (2006), pp. 1-28.

[45] D. D. Suthers, Collaborative representations: Supporting face to face and online knowledge-building discourse, Proc. 34th Hawai' $i$ International Conference on the System Sciences (HICSS-34), January 3-6, 2001, Maui, Hawai' $i$ (CD-ROM), Institute of Electrical and Electronics Engineers, Inc. (IEEE), 2001.

[46] D. D. Suthers, Technology affordances for intersubjective meaning-making: $A$ research agenda for 
CSCL, International Journal of Computers Supported Collaborative Learning, 1 (2006), pp. 315-337.

[47] D. D. Suthers, Towards a systematic study of representational guidance for collaborative learning discourse, Journal of Universal Computer Science, 7 (2001), pp.

http://www.jucs.org/jucs 7 3/towards a systematic study.

[48] D. D. Suthers, N. Dwyer, R. Medina and R. Vatrapu, A framework for eclectic analysis of collaborative interaction, in C. Chinn, G. Erkens and S. Puntambekar, eds., The Computer Supported Collaborative Learning (CSCL) Conference 2007, International Society of the Learning Sciences, New Brunswick, 2007, pp. 694-703.

[49] D. D. Suthers, N. Dwyer, R. Vatrapu and R. Medina, An abstract transcript notation for analyzing interactional construction of meaning in online learning, Proceedings of the 40th Hawai ' $i$ International Conference on the System Sciences (HICSS-40), January 3-6, 2007, Waikoloa, Hawai' $i$ (CD-ROM), Institute of Electrical and Electronics Engineers, Inc. (IEEE), New Brunswick, 2007.

[50] D. D. Suthers and C. Hundhausen, An experimental study of the effects of representational guidance on collaborative learning, Journal of the Learning Sciences, 12 (2003), pp. 183-219.

[51] E. L. Thorndike and R. S. Woodworth, The influence of improvement in one mental function upon the efficiency of other functions, Psychological Review, 9 (1901), pp. 374-382. 